\title{
RECYCLING WASTE NATURAL RUBBER LATEX BY BLENDING WITH POLYSTYRENE - CHARACTERIZATION OF MECHANICAL PROPERTIES
}

\author{
O. BOONDAMNOEN ${ }^{\dagger}$, M. OHSHIMA ${ }^{\text {* }}$, A. R. AZURA ${ }^{\dagger}$, S. CHUAYJULJIT $^{\ddagger}$, A. ARIFFIN $^{\dagger}$ \\ ${ }^{\dagger}$ School of Materials and Mineral Resources Engineering, Engineering Campus, Universiti Sains Malaysia, \\ Nibong Tebal, 14300 Penang, Malaysia \\ ${ }^{s}$ Department of Chemical Engineering, Kyoto University, Nishikyo-ku, Kyoto 615-8510, Japan \\ ${ }^{\ddagger}$ Department of Materials Science, Faculty of Science, Chulalongkorn University, Bangkok 10330, Thailand \\ oshima@cheme.kyoto-u.ac.jp*
}

\begin{abstract}
Waste natural rubber latex was blended with polystyrene (WNRL/PS) for recycling. A mixture with a 50/50 ratio of rubber to PS was blended by an internal mixer (Haake) at $140{ }^{\circ} \mathrm{C}$ and $60 \mathrm{rpm}$. The PS became the matrix, and the WNRL was dispersed within the matrix. Dynamically vulcanized natural rubber/PS (NRv/PS) blends and natural rubber/PS (NR/PS) blends without vulcanization were also prepared in the same way for comparison. The tensile properties and morphologies of all blends were investigated. The crosslinking density was determined by using the Flory-Rehner equation. The experiments showed that the WNRL/PS blend exhibited a higher tensile strength, Young's modulus and crosslinking density, but a lower elongation at break, than the NRv/PS and NR/PS blends. SEM observations of the tensile fracture surface and TEM observations of the blend morphology indicated that the WNRL/PS blend needed a higher energy to break than the NRv/PS and NR/PS blends.
\end{abstract}

Keywords: waste natural rubber latex; crosslinking density; blends; recycling rubber latex.

\section{Introduction}

In Southeast Asia, the consumption of natural rubber latex continues to increase. Industries are expanding production of latex products, such as industrial gloves, prophylactics and others products ${ }^{1}$. In these industries, a high percentage of waste natural rubber latex (WNRL) is formed because of the need to satisfy stringent product quality requirements and the unstable nature of latex. WNRL generally contains high-quality rubber hydrocarbons with minimal crosslinking ${ }^{2-3}$. The industries face economic and environmental challenges if they cannot reuse the WNRL appropriately. In fact, the WNRL has the potential to be reused, which is why many researchers have investigated WNRL. Polystyrene possesses very good processing characteristic and is high strength and low cost; therefore, it was selected for this study. Previous research on recycling of waste natural rubber has focused mainly on filler usage for epoxidized natural rubber latex (ENR). The results have shown that waste latex rubber could be used as an 
economical filler ${ }^{2}$. It has also been reported that waste rubber dust from the textile rubber cots industry could be used to modify waste polypropylene (WPP) ${ }^{4}$.

In this work, an attempt was made to recycle waste natural rubber latex by blending it with polystyrene. A dynamically vulcanized blend was prepared for comparison, and mechanical properties, such as tensile strength, elongation at break and Young's modulus, were studied. The crosslinking density and tensile fracture behavior were investigated via TEM morphology observations.

\section{Experimental}

\subsection{Materials}

Natural rubber (NR) was purchased from Zarm Scientific Sdn. Bhd., Malaysia. The polystyrene (PS) (weight-average molecular weight, Mw, 180,100) used was supplied from Total Petrochemicals, Malaysia. The waste natural rubber latex (WNRL), which is a waste product, was collected from the latex laboratory at the School of Materials and Mineral Resource Engineering, Universiti Sains Malaysia. Only one formula was used for control of the characteristics (Latex $=100 \mathrm{phr}, \mathrm{KOH}=0.3 \mathrm{phr}$, Sulphur $=0.5 \mathrm{phr}$, $\mathrm{ZDEC}=0.75 \mathrm{phr}, \mathrm{ZnO}=0.25 \mathrm{phr}$ and $\mathrm{AnO}=0.5 \mathrm{phr}$ ). All other ingredients (Sulfur, ZDEC, $\mathrm{ZnO}$ and $\mathrm{AnO}$ ) were supplied from Cabot Cans Sdn. Bhd., Malaysia.

\subsection{Preparation of $W N R L$ sheet forms}

The WNRL was cast on a glass plate and dried at ambient temperature for 3 days. Then, it was hung at ambient temperature for further drying. The WNRL sheet was milled by a two-roll mill and cut into small pieces to use as a blend component.

\subsection{Mixing and molding}

The blends were prepared by melt blending WNRL and polystyrene (PS) at a 50/50 weight ratio. The melt blending was performed in a Hake internal mixer (Model Polydrive R600/610) at $140{ }^{\circ} \mathrm{C}$. PS was preheated in the chamber for $1 \mathrm{~min}$ and then treated under a shear rotation to induce melting at a rotor speed of $60 \mathrm{rpm}$ for another 2 min. The WNRL was then added and mixing was continued for $11 \mathrm{~min}$. After mixing, the blend was removed and left at ambient temperature prior to compression. The natural rubber/PS (NR/PS) blend was prepared in the same way.

The dynamically vulcanized natural rubber/PS (NRv/PS) blend was prepared with the same formula as the WNRL/PS blend. The ingredients (Sulfur $=0.5 \mathrm{phr}, \mathrm{ZDEC}=0.75 \mathrm{phr}$, $\mathrm{ZnO}=0.25 \mathrm{phr}$ and $\mathrm{AnO}=0.5 \mathrm{phr}$ ) were the same, and the addition of rubber and mixing were performed in the same manner as for the WNRL/PS blend.

All blends were preheated for $6 \mathrm{~min}$ and compressed for $4 \mathrm{~min}$ at $140{ }^{\circ} \mathrm{C}$. After compression, the sheets were cooled under a pressure of $6.89 \mathrm{MPa}$ for $3 \mathrm{~min}$ at ambient temperature. Samples were cut from the sheet to allow investigation of the mechanical properties. 


\subsection{Characterization}

The sheets were cut into strips with the dimensions 115.0x12.2x1.0 mm (LxWxT). A strip was used instead of a dumbbell shape for tensile testing due to the brittle nature of the samples. The tensile properties were measured using an Autograph (Shimadzu Autographs AGS-J series). The testing was performed according to the ASTM D 412 test method with a crosshead speed $50 \mathrm{~mm} / \mathrm{min}$. The sample mean values were calculated using at least five samples. The crosslinking density was determined by measuring the swelling percentage and applying the Flory-Rehner equation ${ }^{5}$, Eq. 1 . The tensile fracture surfaces were observed using a scanning electron microscope (SEM, Tiny-SEM, Technex, Japan) after a thin gold layer was applied. The morphology of the blends was observed using a JEOL JEM-2000FX. The ultra-thin sample layers were cut using a cryomicrotome, RMC-made Boeckeler, and then stained in $5 \mathrm{ml}$ of $1 \% \mathrm{OsO}_{4}$ solution for $15 \mathrm{~min}$. Equation 1 is defined as follows:

$$
-\ln \left(1-V_{r}\right)-V_{r}-\chi V_{r}^{2}=2 \rho_{R N} V_{o}\left(2 M_{c}\right)^{-1} V_{r}^{1 / 3}
$$

where, $\left(2 \mathrm{M}_{\mathrm{c}}\right)^{-1}$ is the crosslink density, $\chi$ is the interaction parameter between rubber and $2,2,4$ trimethylpentane $(0.49)^{6}, \rho_{\mathrm{RN}}$ is the rubber network density $(0.932)^{7}, \mathrm{~V}_{\mathrm{o}}$ is the molar volume of 2,2,4 trimethylpentane $(165.55 \mathrm{~mol} / \mathrm{ml})$ and $V_{r}$ is the volume fraction of swollen rubber at equilibrium.

\section{Results and discussion}

\subsection{Tensile properties}

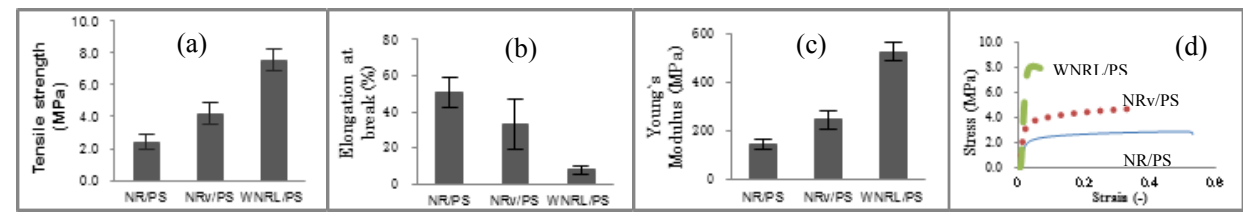

Fig. 1 Tensile properties of the WNRL/PS blends: (a) tensile strength, (b) Elongation at break, (c) Young's Modulus and (d) stress-strain curves

Fig. 1 (a) shows the tensile strength of the different rubber/PS blends. The WNRL/PS blend possessed the highest tensile strength, whereas the NRv/PS and NR/PS blends displayed the next-highest and lowest strengths, respectively. This high strength is because WNRL contains crosslinked molecules formed in the process of latex compounding. The crosslinking connects the rubber chains together and resists failure when a force is applied. As a result, the blends that contained crosslinked molecules had a high tensile strength. For the same reason, the NRv/PS blend exhibited the next-highest tensile strength.

The results of the elongation at break (EB) tests are shown in Fig. 1 (b). The NR/PS blend displayed the highest EB, NRv/PS had the second-highest value and WNRL/PS had the lowest value. This result is reasonable because the crosslinking physically connected the rubber chains and increased the rigidity, but at the same time it prevented the molecule chains from easily moving. Thus, it is expected that the blend that possesses 
fewer crosslinked molecules will show a higher EB. The amount of crosslinking was determined by the crosslinking density, as shown in Table 3 . The table shows that the crosslinking densities of the WNRL/PS and NRv/PS blends were $1.28 \times 10^{-4}$ and $0.52 \times$ $10^{-4} \mathrm{~g}-\mathrm{mol} / \mathrm{cm}^{3}$, respectively, whereas the NR/PS blend was not crosslinked.

The results for Young's modulus were similar to those of tensile strength, as shown in Fig. 1 (c). The order of Young's modulus was WNRL/PS $>$ NRv/PS $>$ NR/PS. Again, this order is due to the presence of crosslinking in the rubber matrix of the WNRL/PS and NRv/PS blends. The higher crosslinking density restricts chain mobility and deformation, which increases the stiffness of the blends ${ }^{8}$.

The ratio of WNRL to PS affects the mechanical properties of the blend. It is predicted that the tensile strength and Young's modulus will decrease, and elongation at break will increase, when the WNRL fraction increases because WNRL reduces the blend stiffness.

By comparing the mechanical properties of the WNRL/PS blend with the dynamically vulcanized NR/PS blend, which used sulfur as a crosslinking agent in earlier research $^{9}$, we determined that the WNRL/PS blend yielded better mechanical properties: higher tensile strength, higher elongation at break and higher Young's modulus. WNRL is a useful additive and can be recycled by blending it with polystyrene.

\subsection{Crosslinking density}

Table 1. Crosslinking density of the blends.

\begin{tabular}{ll}
\hline Sample & Crosslinking density $\left(\mathrm{g}-\mathrm{mol} / \mathrm{cm}^{3}\right)$ \\
\hline NR/PS & \\
NRv/PS & - \\
WNRL/PS & $0.52 \times 10^{-4}$ \\
\hline
\end{tabular}

*Sample was dissolved in solvent according to no crosslinking.

Table 1 shows the crosslinking density in the rubber part of the WNRL and NRv blends. The WNRL blend possesses a higher crosslinking density than the NRv blend, even though the amount of sulfur used in both samples was the same as detailed in sections 2.1 and 2.2. This result could be because the chain lengths of sulfur linkages (crosslinks) were shorter, and the number of the sulfur linkages larger, in the WNRL blend than those in the NRv blend. This could be caused by differences in the reaction, sulfur stabilization and desulfurization times for the two blends. In the vulcanization process, sulfur was used as crosslinking agent, and polysulfide was formed with a high sulfur rank. After the initial crosslinks were formed, the polysulfidic crosslinks were rearranged to a more stable configuration. The polysulfidic crosslinks were broken down, and fragments of the original sulfur crosslinks reformed into new crosslinks between the rubber chains. The new crosslinks may have a lower sulfur rank and a larger number of crosslinking points ${ }^{10-11}$. 


\subsection{Morphology}

Fig. 2 presents SEM images of the tensile fracture surfaces of the NR/PS, NRv/PS and WNRL/PS blends. The fracture surface can elucidate an aspect of the failure, and a rougher fracture surface indicates that a higher energy was needed to break the sample ${ }^{7,12-13}$. This may be explained by crack propagation phenomena: the fracture first forms small cavities in interior of the cross section, and then the crack continues to grow in a direction perpendicular to the stress direction. Crack propagation is resisted by dispersed particles, filler and crosslinks. In the blends, the dispersed particles and crosslinks hinder crack propagation. The NR/PS blend has lower amount of hindrances, namely more slip regions. Thus, the NR/PS fracture surface is smoother compared to the NRv/PS and WNRL/PS blends. These micrographs agree with the tensile strength results because the samples with a rougher fracture surface have a higher tensile strength.
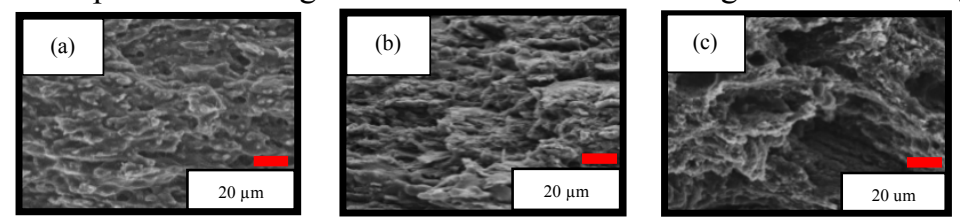

Fig. 2 SEM micrograph of tensile fractures of the blends: (a) NR/PS, (b) NRv/PS and (c) WNRL/PS

The TEM images of different blends are shown in Fig. 3. Due to the $\mathrm{OsO}_{4}$ stain, the light parts indicate PS and the dark parts indicate rubber. It can be clearly seen that phase inversion occurred in the blends as the degree of vulcanization increased. The PS phase transformed from the dispersed phase to co-continuous phase in the NRv/PS blend and eventually it became the matrix phase in the WNRL/PS blend. This observation agrees with the schematic representation of phase inversion in vulcanization blends 9 .
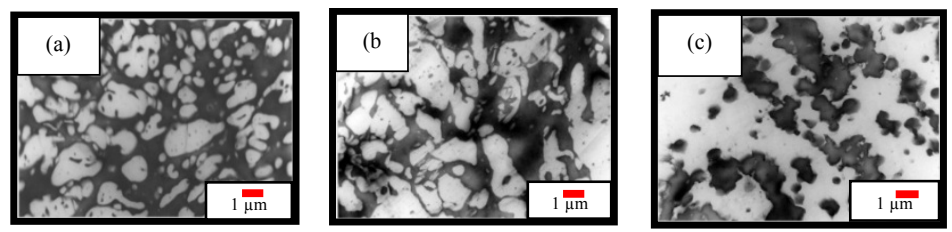

Fig. 3 TEM micrographs of the blends: (a) NR/PS, (b) NRv/PS and (c) WNRL/PS (White: PS, Black: rubber)

The crosslinking in the rubber phase connects the polymer chains tightly, which increases the surface tension and viscosity and makes the phase more difficult to disperse. Generally, the lower viscosity component can be easily dispersed in the blends, as can be seen in Fig. 4 (a). However, in case of the blends that contain crosslinking ${ }^{14}$, a higher crosslinking density makes the viscosity higher and elongation more difficult. Thus, the continuous rubber phase was broken into smaller particles under stress.

Fig. 3 (b) and (c) also show that the rubber particle size decreases as the crosslinking density increases. Earlier research showed that a higher degree of crosslinking results in smaller and more abundant particles ${ }^{14}$. Nevertheless, the WNRL/PS blend exhibited agglomeration of rubber particles, which was observed in the SEM micrograph in Fig. 2 (c), where the holes represent the detachment of WNRL. Changing the volume 
percentage of WNRL in the blend could increase the tensile strength, but the presence of large, dispersed WNRL domains drastically reduced the elongation at break. Therefore, the optimum blend ratio of WNRL is likely higher than $50 \%$, which would preserve the WNRL matrix and allow practical use of the recycled WNRL/PS blend.

\section{Conclusion}

The potential to reuse waste natural rubber latex was investigated. Blending waste natural rubber with polystyrene improved the Young's modulus but reduced the elongation at break. The mechanical properties of the WNRL/PS blend showed better results compared to blends of conventional thermoplastics based on natural rubber and polystyrene. Phase inversion of the blend occurs when the WNRL/PS ratio is 50/50. The WNRL/PS blend exhibited the highest yield stress. However, the WNRL/PS blend displayed lower plasticity due to agglomeration of rubber particles. Additional work is needed to determine the optimal blend ratio for the WNRL/PS system. At this stage, however, it can be said that WNRL can be recycled to create a material that is both economical and ecologically friendly.

\section{Acknowledgments}

The authors thank the AUN/SEED Net Project (Grant no. 304/6050170) for financial support. They also gratefully acknowledge the School of Materials and Mineral Resources Engineering, Universiti Sains Malaysia and Materials and Processing Engineering, Kyoto University for facility support.

\section{References}

1. Rajan V.V., Dierkes W.K., Joseph R. and Noordermeer J.W.M., Prog.Polym.Sci. 31, 811-834 (2006).

2. Mathew G., Singh R.P., Nair N.R. and Sabu T, Polymer 42, 2137-2165 (2001)

3. Rajalekshmi S. and Rani J., Journal of Elastomers and Plastics 34, 313-322 (2002)

4. Jose J., Satapathy S., Nag A. and Nando G. B., Process Saf. Environ. Prot. 85, 318-326 (2007)

5. George J., Varughese K. T. and Thomas S., Polymer 41, 1507-1517 (2000)

6. Robert A., Orwoll and Arnold P. A. Physical Properties of Polymer Hanbook. p. 188 (1996)

7. Ismail H., Nordin R. and Noor A. M., Iranian Polymer J 12, 373-380 (2003)

8. Awang M. and Ismail H., Polym. Test. 27, 321-329 (2008)

9. Asaletha R., Kumaran M. G. and Thomas S., Eur. Polym. J. 35, 253-271 (1999)

10. Prasenjeet G., Santhoji K., Priyan P., James M. C., Venkat V. and Kenneth A. W., Rubber Chemistry \& Technology 76, 592-693 (2003)

11. Gary R. H., Materials and Compounds, Engineering with Rubber: How to Design Rubber Components (New York, 1992), p. 19-23

12. Teh P. L., Ishak Z. A. M., Hashim A. S., Karger-Jocsis J. and Ishiaku U. S., Eur. Polym. J. 40, 2513-2521 (2004)

13. Ismail H., Suryadiansyah., Polymer-Plastics Technology and Engineering 41, 833-845 (2002)

14. Ghosh P., Bhattopadhyay and Sen A. K., Polymer 35, 3958-3965 (1994)

15. Gorage J., Varughese K.T. and Thomas S., Polymer 41, 1507-1517 (1999) 